\title{
Research
}

\section{Academic Motivation and Psychological Needs as Predictors of Suicidal Risk}

\author{
Jerin Lee, Edward C. Chang, Abigael G. Lucas, and Jameson K. Hirsch
}

\begin{abstract}
This study examined academic motivation and basic psychological needs as predictors of suicidal risk (namely, depressive symptoms and suicidal behaviors) in a sample of 348 college students. Results from regression analyses indicated that academic motivation was a significant predictor of suicidal risk. The inclusion of basic psychological needs significantly augmented the prediction model. The authors discuss implications for considering academic motivation and basic psychological needs in college counseling on the basis of the results.
\end{abstract}

Keywords: academic motivation, basic psychological needs, suicidal risk, depressive symptoms, suicidal behaviors

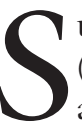

uicide represents a serious mental health concern faced by college students (Schwartz \& Friedman, 2009; Westefeld et al., 2006). Among college-age adults, suicide is the second leading cause of death, behind unintentional injury (Centers for Disease Control and Prevention, 2014). According to the model proposed by Bonner and Rich (1987), both distal (i.e., depressive symptoms) and proximal (i.e., suicidal behaviors) variables are believed to increase the risk of death by suicide in college students. Indeed, although distal risk factors may be insufficient on their own for suicide to occur, they are pertinent to the occurrence of suicide (Mościcki, 1997). Conversely, although proximal risk factors are the most closely related to the suicidal event itself (e.g., suicidal behaviors), they are neither necessary nor sufficient for suicide to occur. When combined, distal and proximal risk factors provide the necessary and sufficient conditions for suicide, making the examination of both in studies of suicidal risk particularly valuable. Consistent with this framework, findings from numerous studies have implicated depressive symptoms and suicidal behaviors (e.g., suicide ideation) as significant risk factors associated with suicide in college student populations (e.g., Dvorak, Lamis, \& Malone, 2013; Farabaugh et al., 2012). Thus, given the seriousness of suicide and its prevalence in college-age adults (Schwartz \& Friedman, 2009), it is not surprising that researchers have become interested in identifying predictors of suicidal risk in college student populations.

Given that pressures specific to the academic environment often accompany the college experience (e.g., academic achievement, continuous academic evaluation;

Jerin Lee, Edward C. Chang, and Abigael G. Lucas, Department of Psychology, University of Michigan; Jameson K. Hirsch, Department of Psychology, East Tennessee State University. This work was completed in partial fulfillment of the requirements for the accelerated master's degree program in psychology by the first author under the guidance of the second author. The authors would like to thank members of the Perfectionism and Optimism-Pessimism Lab at the University of Michigan for their support and suggestions on earlier drafts of this article. Correspondence concerning this article should be addressed to Jerin Lee or Edward C. Chang, Department of Psychology, University of Michigan, 530 Church Street, Ann Arbor, MI 48109 (email: jerinlee@umich.edu or changec@umich.edu).

(C) 2019 by the American Counseling Association. All rights reserved. 
Ross, Niebling, Heckert, 1999; Smith, Langenbacher, Kudlac, \& Fera, 2013), one construct that is particularly important to consider is the need to achieve in the academic context-namely, academic motivation. Academic motivation is defined as a set of motives associated with diverse achievement goals (e.g., intrinsic motivation, extrinsic motivation; Vallerand et al., 1992). Findings from studies on academic motivation have shown it to be associated with academic performance (e.g., Meriac, 2015) and psychological adjustment (e.g., Baker, 2004; Miquelon, Vallerand, Grouzet, \& Cardinal, 2005), including indices of suicidal risk (e.g., depressive symptoms, suicidal behaviors; Klibert, Langhinrichsen-Rohling, Luna, \& Robichaux, 2011; Wang, 2012). For example, in a study of 475 college students, Klibert et al. (2011) found that greater motivation for academic achievement was associated with less suicidal thoughts and behaviors. Accordingly, it is reasonable that academic motivation would play an important role in suicidal risk among college students.

However, the college experience often involves personal and social growth, and it becomes equally as important to see how motives beyond those associated with the academic context might also be involved in suicidal risk. Indeed, other researchers have argued that broader motives may play an important role in psychological adjustment. According to Ryan and Deci (2000a), basic psychological needs represent fundamental human motives that are predicated on three dimensions: autonomy, defined as the experience of personal choice in one's behavior; competence, defined as the ability to experience mastery and effectively bring about desired outcomes; and relatedness, defined as feelings of closeness and connectedness with significant others. Furthermore, Ryan and Deci (2000a) proposed that the fulfillment of these needs can contribute to growth and well-being. Conversely, when these needs are threatened, an individual can experience adverse outcomes, such as depressive or anxious symptomatology. Consistent with this notion, studies have shown that the inability to meet these needs is associated with higher suicidal risk in college students (Britton, Van Orden, Hirsch, \& Williams, 2014; Schiffrin et al., 2014). For example, in a sample of 439 college students, Rowe, Walker, Britton, and Hirsch (2013) found that basic psychological needs were negatively correlated with both depressive symptoms and suicidal behaviors. However, no study to date has examined whether basic psychological needs continue to represent important predictors of suicidal risk in college students, beyond the role of a more contextspecific motive such as academic motivation.

\section{Purpose of the Study}

The purpose of the present study was to examine the extent to which basic psychological needs might add to the prediction of suicidal risk beyond academic motivation in a college student population. Consistent with past research (e.g., Miquelon et al., 2005), we expected academic motivation to be an important predictor of both depressive symptoms and suicidal behaviors. Additionally, consistent with Deci and Ryan's (2000) contention that basic psychological needs represent a broader set of fundamental human motives and with previous researchers' (Rowe et al., 2013; Schiffrin et al., 2014) linking of basic psychological needs to suicidal risk, we predicted that the inclusion of basic psychological needs would account 
for a significant amount of additional variance in suicidal risk. Furthermore, given that studies on suicidal risk have pointed to the specific importance of being interconnected with others (Baumeister \& Leary, 1995; Van Orden et al., 2010), we hypothesized that the inability to meet relatedness needs would play a particularly important role in predicting suicidal risk in college students.

\section{Method}

\section{Participants}

College students ( $N=348$ ) were recruited from a university in the southeastern United States. The majority of the sample was female $(n=229)$, followed by male $(n=113)$, transgender $(n=3)$, and no response $(n=3)$. Participants' ages ranged between 18 and 58 years, with a mean of $21.8(S D=5.27)$. Most participants indicated that they were European American (85.9\%), followed by African American (5.7\%), no response (3.4\%), Asian American (3.2\%), and Latino American (1.7\%). Because there were a few incomplete responses across the present study measures, sample sizes varied across analyses. Sample sizes for each measure are provided in Table 1.

\section{Measures}

Academic motivation. Academic motivation was assessed with the Academic Motivation Scale (AMS; Vallerand et al., 1992), a 28-item measure consisting of seven subscales examining intrinsic motivation, extrinsic motivation, and amotivation. For the present study, the Amotivation subscale was not used given that a lack of motivation represents a common symptom of depression (Stahl, 2002). The three subscales of intrinsic motivation are Intrinsic Motivation to Know (e.g., "Because I experience pleasure and satisfaction while learning new things"), Intrinsic Motivation to Achieve (e.g., "For the pleasure

TABLE 1

\section{Correlations, Means, and Standard Deviations for Study Measures in College Students}

\begin{tabular}{|c|c|c|c|c|}
\hline \multirow[b]{2}{*}{ Measure } & \multicolumn{2}{|c|}{ Correlation } & \multirow[b]{2}{*}{$M$} & \multirow[b]{2}{*}{$S D$} \\
\hline & DS $(n=341)$ & SB $(n=334)$ & & \\
\hline Intrinsic motivation to know & $-.13^{*}$ & -.05 & 13.86 & 3.49 \\
\hline Intrinsic motivation to achieve & -.08 & -.01 & 12.70 & 3.57 \\
\hline Intrinsic motivation to experience stimulation & .01 & .06 & 10.95 & 3.76 \\
\hline Extrinsic identified regulation & $-.21^{\star \star *}$ & $-.24^{\star \star *}$ & 15.25 & 3.40 \\
\hline Extrinsic introjected regulation & -.04 & -.02 & 14.01 & 3.64 \\
\hline Extrinsic external regulation & $-.13^{*}$ & $-.15^{\star *}$ & 15.29 & 3.42 \\
\hline Competence & $-.53^{\star \star \star}$ & $-.34^{\star \star *}$ & 29.02 & 5.98 \\
\hline Autonomy & $-.47^{\star \star \star}$ & $-.34^{\star * *}$ & 32.79 & 6.07 \\
\hline Relatedness & $-.47^{\star \star \star}$ & $-.40^{\star \star *}$ & 42.02 & 8.40 \\
\hline$M$ & 10.64 & 2.24 & & \\
\hline$S D$ & 11.41 & 3.30 & & \\
\hline
\end{tabular}

Note. Sample sizes varied across analyses because of incomplete responses. DS = depressive symptoms; $\mathrm{SB}=$ suicidal behavior.

${ }^{\star} p<.05 .{ }^{* *} p<.01 .{ }^{* * *} p<.001$. 
I experience while surpassing myself in my studies"), and Intrinsic Motivation to Experience Stimulation (e.g., "For the intense feelings I experience when I am communicating my own ideas to others"). The three subscales of extrinsic motivation are Extrinsic Identified Regulation (e.g., "Because I think that a college education will help me better prepare for the career I have chosen"), Extrinsic Introjected Regulation (e.g., "To prove to myself that I am capable of completing my college degree"), and Extrinsic External Regulation (e.g., "In order to obtain a more prestigious job later on"). Respondents are asked to rate the extent to which each item corresponds to the reasons why they are in college on a 7-point Likert-type scale ranging from 1 (does not correspond at all) to 7 (corresponds exactly). The overall internal reliability of the AMS was .88. Evidence for the validity of the AMS has been reported by Vallerand et al. (1993). Higher scores indicate greater intrinsic motivation or extrinsic motivation. For the present study, all six of the intrinsic motivation and extrinsic motivation subscale scores were included as a set in the analyses.

Basic psychological needs. Basic psychological needs were assessed with the General Basic Psychological Needs Scale (GBPNS; Gagné, 2003), a 21 -item self-report measure consisting of three separate subscales: Competence (e.g., "People I know tell me I am good at what I do"), Autonomy (e.g., "I feel like I am free to decide for myself how to live my life"), and Relatedness (e.g., "I get along with people I come into contact with"). Respondents are asked to rate how true or untrue they found each item to be in relation to themselves on a 7-point Likerttype scale ranging from 1 (not at all true) to 7 (very true). The overall internal reliability of the GBPNS was .89. Evidence for the validity of the GBPNS has been reported by Johnston and Finney (2010). Higher scores on the subscales indicate greater needs satisfaction. For the present study, all three of the subscale scores were included as a set in the analyses.

Suicidal risk. To assess for suicidal risk, we used the Beck Depression Inventory (BDI; Beck, Ward, Mendelson, Mock, \& Erbaugh, 1961) and the Suicidal Behaviors Questionnaire-Revised (SBQ-R; Osman et al., 2001). The BDI is a commonly used 21 -item self-report measure of depressive symptoms. Respondents are asked to rate the extent to which they have experienced specific depressive symptoms in the past week across a 4-point Likert-type scale (e.g., $0=I$ do not feel sad to 3 $=I$ am so sad or unbappy that I can't stand it). The internal reliability of the BDI was .95. Evidence for the validity of the BDI has been reported by Beck et al. (1961). Higher scores on the BDI indicate greater symptoms of depression. For the present study, the total score of the BDI was used in the analyses.

The SBQ-R is a four-item measure that assesses key aspects of suicidal behaviors, including lifetime ideation and/or suicide attempt ("Have you ever thought about or attempted to kill yourself?"), frequency of suicide ideation over the past 12 months ("How often have you thought about killing yourself in the past year?"), threat of suicide attempt ("Have you ever told someone that you were going to commit suicide or that you might do it?"), and likelihood of suicidal behavior in the future ("How likely is it that you will attempt suicide someday?"). The responses for each item are measured across a 5- to 7-point Likert-type scale, for example, ranging from 0 or 1 (never) to 5 (very often) or 6 (very likely). The internal reliability of 
the SBQ-R was .81. Evidence for the validity of the SBQ-R has been reported by Osman et al. (2001). Higher scores on the SBQ-R indicate greater suicidal behaviors. For the present study, the total score of the SBQ-R was used in the analyses.

\section{Procedure}

Approval for the study was obtained from the institutional review board prior to data collection. Participants were recruited through an electronic data collection system that allows potential participants to voluntarily enroll in the studies they would like to participate in. Upon completion of the survey, participants received either course-required credit or extra credit. Participants completed surveys online in a location of their choosing after first clicking an electronic informed consent. The survey took approximately 30 minutes to complete. Given that survey answers were untraceable to the identity of the participant, we were unable to follow up with those who may identify as being at risk for suicide.

\section{Data Analyses}

Prior to recruiting participants, we expected some of the associations to be in the medium effect size range $(r=.30)$. Accordingly, results from an a priori analysis for achieving a power of .95 to detect a medium effect indicated that we needed a minimum of 134 participants $(p<.05$, two-tailed). Given that we were able to recruit 348 college students for the study, we believe that our sample size was appropriate for the subsequent data analyses.

To determine whether basic psychological needs added, beyond academic motivation, to the prediction of suicidal risk in college students, we conducted a set of hierarchical regression analyses. In these analyses, demographic variables (sex and ethnicity) were entered in Step 1, followed by scores on the six academic motivation subscales (as a set) in Step 2, then by scores on the three basic psychological needs subscales (as a set) in Step 3. To determine whether any of the predictors accounted for a small, medium, or large amount of the variance in suicidal risk, we used Cohen's (1977) convention for small $\left(f^{2}=.02\right)$, medium $\left(f^{2}=.15\right)$, and large effects $\left(f^{2}=.35\right)$ as a general guide.

Additionally, we were curious to see whether academic motivation and basic psychological needs would remain essential predictors of suicidal behaviors, even after controlling for concurrent depressive symptoms (e.g., Klibert et al., 2011). We conducted a supplementary regression analysis in which we included scores on demographic variables in Step 1, followed by depressive symptoms in Step 2, then scores on academic motivation in Step 3, and finally scores on basic psychological needs in Step 4.

\section{Results}

Correlations involving academic motivation and basic psychological needs with each of the suicidal risk indices are presented in Table l. Intrinsic motivation to know was negatively associated with depressive symptoms $(r=-.13, p<.05)$. 
Extrinsic identified regulation was negatively associated with depressive symptoms $(r=-.21, p<.001)$ and suicidal behaviors $(r=-.24, p<.001)$. Extrinsic external regulation was also negatively associated with depressive symptoms $(r$ $=-.13, p<.05)$ and suicidal behaviors $(r=-.15, p<.01)$. Each of the basic psychological needs was negatively associated with both indices of suicidal risk.

Results of regression analyses for depressive symptoms and suicidal behaviors are presented in Table 2. For depressive symptoms, demographics was not found to account for a significant amount of variance, $F(2,325)=1.16$, ns. However, academic motivation accounted for a small $\left(f^{2}=.08\right)$ but significant $8 \%$ of variance, $F(6,319)=4.49, p<.001$. Within the academic motivation set, extrinsic identified regulation $(\beta=-.27)$ and extrinsic introjected regulation $(\beta=.2 \mathrm{l})$ significantly predicted depressive symptoms. Beyond academic motivation, basic psychological needs accounted for a large $\left(f^{2}=.35\right) 26 \%$ of additional variance in depressive symptoms, $F(3,316)=41.72, p<.001$. Within this set, competence ( $\beta=-.34)$ and autonomy $(\beta=-.20)$ were found to be significant predictors. The

\section{TABLE 2}

\section{Results of Hierarchical Regression Analyses Showing Amount of Variance in Indices of Suicidal Risk Accounted for by Academic Motivation and Basic Psychological Needs in College Students}

\begin{tabular}{|c|c|c|c|c|}
\hline Outcome and Predictor & $\beta$ & $R^{2}$ & $\Delta \boldsymbol{R}^{2}$ & $\boldsymbol{F}$ \\
\hline \multicolumn{5}{|l|}{ Depressive symptoms $(n=327)$} \\
\hline Step 1: Demographics & & .01 & & 1.16 \\
\hline Sex & -.08 & & & \\
\hline Race & -.01 & & & \\
\hline Step 2: Academic motivation & & .08 & .08 & $4.49^{\star \star \star}$ \\
\hline Intrinsic motivation to know & -.12 & & & \\
\hline Intrinsic motivation to achieve & -.07 & & & \\
\hline Intrinsic motivation to experience stimulation & .07 & & & \\
\hline Extrinsic identified regulation & $-.27^{\star *}$ & & & \\
\hline Extrinsic introjected regulation & $.21^{*}$ & & & \\
\hline Extrinsic external regulation & -.03 & & & \\
\hline Step 3: Basic psychological needs & & .34 & .26 & $41.72^{\star \star \star}$ \\
\hline Competence & $-.34^{\star \star \star}$ & & & \\
\hline Autonomy & $-.20^{\star \star}$ & & & \\
\hline \multirow{2}{*}{\multicolumn{5}{|c|}{ Suicidal behaviors $(n=331)$}} \\
\hline & & & & \\
\hline Step 1: Demographics & & .01 & & 1.04 \\
\hline Sex & .05 & & & \\
\hline Race & -.06 & & & \\
\hline Step 2: Academic motivation & & .09 & .08 & $4.86^{\star \star \star}$ \\
\hline Intrinsic motivation to know & .00 & & & \\
\hline Intrinsic motivation to achieve & .00 & & & \\
\hline Intrinsic motivation to experience stimulation & .03 & & & \\
\hline Extrinsic identified regulation & $-.35^{\star \star \star}$ & & & \\
\hline Extrinsic introjected regulation & $.20^{*}$ & & & \\
\hline Extrinsic external regulation & -.02 & & & \\
\hline Step 3: Basic psychological needs & & .20 & .11 & $14.68^{\star * *}$ \\
\hline Competence & -.06 & & & \\
\hline Autonomy & $-.12^{\dagger}$ & & & \\
\hline Relatedness & $-.24^{\star \star}$ & & & \\
\hline
\end{tabular}

${ }^{\dagger} p<.10 .{ }^{*} p<.05 .{ }^{* \star} p<.01 .{ }^{\star \star *} p<.001$. 
total prediction model was found to account for a large $\left(f^{2}=.52\right) 34 \%$ of the variance in depressive symptoms, $F(11,316)=15.07, p<.001$.

Next, for suicidal behaviors, demographics was again not found to account for a significant amount of variance, $F(2,329)=1.04$, ns. However, academic motivation accounted for a small $\left(f^{2}=.09\right)$ but significant $8 \%$ of variance, $F(6,323)=4.86, p<.001$. Within the academic motivation set, extrinsic identified regulation $(\beta=-.35)$ and extrinsic introjected regulation $(\beta=.20)$ significantly predicted suicidal behaviors. Beyond academic motivation, basic psychological needs accounted for a small $\left(f^{2}=.12\right)$ but significant $11 \%$ of additional variance in suicidal behaviors, $F(3,320)=14.68, p<.001$. Within the basic psychological needs set, autonomy $(\beta=-.12)$ and relatedness $(\beta=$ $-.24)$ emerged as significant predictors. The total prediction model was found to account for a medium $\left(f^{2}=.25\right) 20 \%$ of the variance in suicidal behaviors, $F(11,320)=7.22, p<.001$.

Finally, results of an additional regression analysis for predicting suicidal behaviors after controlling for concurrent depressive symptoms indicated that both academic motivation (i.e., extrinsic identified regulation; $\beta=-.22$, $p<.01$ ) and basic psychological needs (i.e., relatedness; $\beta=-.20, p=.01$ ) remained essential predictors of suicidal behaviors. Overall, the inclusion of basic psychological needs as a set was consistently found to augment the prediction model of suicidal risk, even after accounting for the variance in suicidal risk associated with academic motivation.

\section{Discussion}

We conducted the present study to determine whether broader motivesnamely, basic psychological needs-would further contribute to the prediction of suicidal risk in college students beyond academic motivation. Consistent with expectations, our results indicated that academic motivation, as a set, was a significant predictor of both depressive symptoms and suicidal behaviors in college students. It is interesting to note that extrinsic introjected regulation was found to be a unique positive predictor of depressive symptoms and suicidal behaviors, whereas extrinsic identified regulation was found to be a unique, and slightly more robust, negative predictor of both indices of suicidal risk. According to researchers who study the role of motivation on adjustment (e.g., Ryan \& Deci, 2000b; Vallerand et al., 1992), extrinsic introjected regulation involves a point across the self-determination spectrum at which individuals begin to internalize reasons why they do things but are ultimately motivated to maintain self-esteem or avoid guilt. In contrast, extrinsic identified regulation involves the subsequent point at which individuals have completely internalized a motive and view the motive as being valuable for the self. Thus, not only do our findings indicate that the partial internalization of motives may play an important role in the development of depressive symptoms and suicidal behaviors, but they also demonstrate that the inability to find personal value in one's motives for doing things (i.e., extrinsic identified motivation) may be particularly detrimental to students. In other words, a student who studies 
because that is what he or she believes a good student should do would be at greater risk for suicide than a student who studies because he or she believes that it is personally valuable and important.

Expanding on past research findings showing that basic psychological needs are essential predictors of suicidal risk (e.g., Rowe et al., 2013; Schiffrin et al., 2014), we found that basic psychological needs, as a set, further augmented the prediction of depressive symptoms and suicidal behaviors in college students, above and beyond academic motivation. However, in contrast to expectations that relatedness would predict both indices of suicidal risk, the inability to meet autonomy needs was found to be a significant predictor of depressive symptoms and suicidal behaviors. Moreover, the inability to meet competence needs was found to be a significant predictor of depressive symptoms, whereas the inability to meet relatedness needs was found to be a significant predictor of suicidal behaviors. Not only do these findings suggest that the inability to feel a sense of free will when taking action (i.e., autonomy) may play a particularly important role in contributing to suicidal risk, but they also demonstrate that the potential consequences associated with an inability to meet different basic psychological needs might be specific to different psychological outcomes. For example, whereas the inability to achieve meaningful relations with others might play a more central role in proximal indices of suicidal risk (e.g., suicide ideation, suicide attempts; Van Orden et al., 2010), problems in achieving a sense of mastery might play a more central role in distal indices of suicidal risk (e.g., depressive symptoms; Colman et al., 2011).

Noteworthy results from the additional analysis examining whether academic motivation and basic psychological needs remain essential predictors of suicidal behaviors after controlling for concurrent depressive symptoms indicated a pattern that was comparable with the one found without controlling for depressive symptoms. Exceptions were that extrinsic introjected regulation and autonomy no longer remained significant predictors of suicidal behaviors, which suggests that the effects of extrinsic introjected regulation and autonomy may be specific to distal variables of suicidal risk (e.g., depressive symptoms). Thus, researchers might want to consider controlling for distal variables of suicidal risk when examining suicidal risk in relation to extrinsic introjected regulation and autonomy.

\section{Implications for College Counseling}

This study's findings offer some important implications for college counselors in potentially minimizing the development or progression of suicidal risk in college students. Our findings regarding the differential associations between dimensions of academic motivation and indices of suicidal risk suggest the value in assessing for academic motivation using all six intrinsic and extrinsic dimensions to best identify which motivational dimensions to facilitate or discourage. For example, given that extrinsic introjected regulation was found to predict both depressive symptoms and suicidal behaviors, it may be important for college counselors to assist students in effectively managing different, and sometimes competing, motives (e.g., internal vs. external motives; Tomiyama \& Mann, 2008) to decrease potential suicidal risk. Indeed, consistent with the finding that nearly $75 \%$ of incoming college students have indicated that they were attending college to get 
a better job or to make more money (Pryor, Hurtado, Sharkness, \& Kom, 2008) and that vocational decision-making processes have been found to be associated with experiences of anxiety in college students (Hawkins, Bradley, \& White, 1977), our findings suggest that college counselors should be aware that an overemphasis on vocational outcomes of higher education (e.g., economic and job security) may be harmful to college students' well-being (Pisarik, 2009). That said, college counselors can help students who may feel pressured to attend college to identify academic pursuits and courses that correspond with their personal values (Scheel, Madabhushi, \& Backhaus, 2009). It may be helpful for researchers to consider the interplay of academic motives and vocational decision-making processes in predicting suicidal risk in college students.

Relatedly, our finding that extrinsic identified regulation predicted suicidal behaviors, even after controlling for concurrent depressive symptoms, points both to the general value of working with students to reduce any concurrent experience of depressive symptoms and to the specific value of providing students with effective strategies (e.g., positive reframing) to cope when they are not able to achieve an outcome that has become personally valued. Our findings suggest the potential value of enhancing students' identification with their academic work to thwart the development of depressive symptoms and/or suicidal behaviors. College counselors can encourage the sustainment of identified motivation by guiding students to engage in activities that facilitate their achievement of selfdefined goals and checking if students' goals continue to be genuinely meaningful to them (Burton, Lydon, D'Alessandro, \& Koestner, 2006).

The findings of this study suggest the fundamental role that basic psychological needs (or lack thereof) play in contributing to suicidal risk beyond academic motivation. For example, working with students to help them develop more effective ways to achieve a general sense of competence and autonomy in their lives might help reduce their risk of developing symptoms of depression. More specifically, college counselors can use motivational interviewing techniques such as affirming and validating students' existing ideas and efforts to foster competence, as well as emphasizing personal choice to support autonomy (Markland, Ryan, Tobin, \& Rollnick, 2005). Additionally, counselors can inform students of courses that provide optimally challenging learning opportunities alongside appropriate feedback (i.e., scaffolding; Urdan \& Schoenfelder, 2006) and implement autonomy-supportive teaching practices (e.g., fostering students' perceptions of having a choice; Niemiec \& Ryan, 2009).

Finally, our results indicate that college students may benefit from opportunities to develop meaningful relationships that foster feelings of relatedness. College counselors can use interventions (e.g., subtle attitude-change strategies) to encourage positive interpretations of social adversity (Walton \& Cohen, 2011) and focus on enhancing students' development of friendships (Pittman \& Richmond, 2008) and quality student-faculty interactions. Furthermore, counselors can direct students to courses that emphasize participation and interaction with classmates (Freeman, Anderman, \& Jensen, 2007), such as through small-group work (Nikolai, Silva, \& Walters, 2017), to help students foster a greater sense of relatedness on campus. Overall, it is apparent that college counselors can play a crucial role in lowering 
suicidal risk by guiding students to understand their academic motivation and basic psychological needs and informing students of resources to help manage their academic motivation and fulfillment of their basic psychological needs.

\section{Limitations}

Despite the importance of the present findings, some limitations are worth noting. First, because our sample was predominantly European American, it would be important to determine if similar or different findings emerge with other ethnic or racial groups. Second, given the cross-sectional design of the present study, it would be useful for future studies to examine the extent to which academic motivation and basic psychological needs satisfaction predict changes in suicidal risk across time. Third, the present study used a convenience sample, self-report measures, and measures of suicidal risk to assess clinical levels; thus, it is important to note that our sample was not representative of a population at high risk for suicide. It would be valuable for future studies to examine samples that may be at high risk for suicide. Fourth, the inclusion of demographics, academic motivation, and basic psychological needs was found to contribute to only $20 \%$ to $34 \%$ of the variance in suicidal risk indices. This large amount of remaining variance indicates the need for additional screening, assessments, and research that will further the understanding of how, and to what extent, other variables might influence suicidal risk among college student populations. Finally, although the implications are written primarily for college counselors, this does not preclude the potential utility the implications may have for academic counselors and advisers in reducing suicidal risk among college students.

\section{Conclusion}

We conducted the present study to determine whether, beyond academic motivation, basic psychological needs represent an important predictor of suicidal risk - namely, depressive symptoms and suicidal behaviors - in college students. Consistent with expectations, we found that both academic motivation and basic psychological needs represent significant predictors of suicidal risk in students. Different dimensions of basic psychological needs were found to additively predict, beyond different facets of academic motivation, depressive symptoms and suicidal behaviors. Overall, our findings highlight the importance of considering the role of specific as well as broad motivational processes for understanding and counseling college student populations that are at risk for suicide.

\section{References}

Baker, S. R. (2004). Intrinsic, extrinsic, and amotivational orientations: Their role in university adjustment, stress, well-being, and subsequent academic performance. Current Psychology, 23, 189-202. doi:10.1007/s12144-004-1019-9

Baumeister, R. F., \& Leary, M. R. (1995). The need to belong: Desire for interpersonal attachments as a fundamental human motivation. PsychologicalBulletin, 117,497-529. doi:10.1037/0033-2909.117.3.497

Beck, A. T., Ward, C. H., Mendelson, M., Mock, J., \& Erbaugh, J. (1961). An inventory for measuring depression. Archives of General Psychiatry, 4, 561-571. doi:10.1001/archpsyc.1961.01710120031004 
Bonner, R. L., \& Rich, A. R. (1987). Toward a predictive model of suicidal ideation and behavior: Some preliminary data in college students. Suicide and Life-Threatening Behavior, 17, 50-63. doi:10.1111/j.1943-278X.1987.tb00061.x

Britton, P. C., Van Orden, K. A., Hirsch, J. K., \& Williams, G. C. (2014). Basic psychological needs, suicidal ideation, and risk for suicidal behavior in young adults. Suicide and Life-Threatening Behavior, 44, 362-371. doi:10.1111/sltb.12074

Burton, K. D., Lydon, J. E., D'Alessandro, D. U., \& Koestner, R. (2006). The differential effects of intrinsic and identified motivation on well-being and performance: Prospective, experimental, and implicit approaches to self-determination theory. Journal of Personality and Social Psychology, 91, 750-762. doi:10.1037/0022-3514.91.4.750

Centers for Disease Control and Prevention. (2014). Leading causes of death reports, national and regional, 1999-2014. Retrieved from http://webappa.cdc.gov/sasweb/ncipc/leadcaus10_us.html

Cohen, J. (1977). Statistical power analysis for the behavioral sciences (Rev. ed.). Hillsdale, NJ: Erlbaum.

Colman, I., Naicker, K., Zeng, Y., Ataullahjan, A., Senthilselvan, A., \& Patten, S. B. (2011). Predictors of long-term prognosis of depression. Canadian Medical Association Journal, 183, 1969-1976. doi:10.1503/cmaj.110676

Deci, E. L., \& Ryan, R. M. (2000). The "what" and "why" of goal pursuits: Human needs and the selfdetermination of behavior. Psychological Inquiry, 11, 227-268. doi:10.1207/S15327965PLI1104_01

Dvorak, R. D., Lamis, D. A., \& Malone, P. S. (2013). Alcohol use, depressive symptoms, and impulsivity as risk factors for suicide proneness among college students. Journal of Affective Disorders, 149, 326-334. doi:10.1016/j.jad.2013.01.046

Farabaugh, A., Bitran, S., Nyer, M., Holt, D. J., Pedrelli, P., Shyu, I., . . Fava, M. (2012). Depression and suicidal ideation in college students. Psychopathology, 45, 228-234. doi:10.1159/000331598

Freeman, T. M., Anderman, L. H., \& Jensen, J. M. (2007). Sense of belonging in college freshmen at the classroom and campus levels. Journal of Experimental Education, 75, 203-220.

Gagné, M. (2003). The role of autonomy support and autonomy orientation in prosocial behavior engagement. Motivation and Emotion, 27, 199-223. doi:10.1023/A:1025007614869

Hawkins, J. G., Bradley, R. W., \& White, G. W. (1977). Anxiety and the process of deciding about a major and vocation. Journal of Counseling Psychology, 24, 398-403. doi:10.1037/0022-0167.24.5.398

Johnston, M. M., \& Finney, S. J. (2010). Measuring basic needs satisfaction: Evaluating previous research and conducting new psychometric evaluations of the Basic Needs Satisfaction in General Scale. Contemporary Educational Psychology, 35, 280-296. doi:10.1016/j.cedpsych.2010.04.003

Klibert, J., Langhinrichsen-Rohling, J., Luna, A., \& Robichaux, M. (2011). Suicide proneness in college students: Relationships with gender, procrastination, and achievement motivation. Death Studies, 35, 625-645. doi:10.1080/07481187.2011.553311

Markland, D., Ryan, R. M., Tobin, V. J., \& Rollnick, S. (2005). Motivational interviewing and self-determination theory. Journal of Social and Clinical Psychology, 24, 811-831. doi:10.1521/jscp.2005.24.6.811

Meriac, J. P. (2015). Examining relationships among work ethic, academic motivation and performance. Educational Psychology, 35, 523-540. doi:10.1080/01443410.2014.895291

Miquelon, P., Vallerand, R. J., Grouzet, F. M., \& Cardinal, G. (2005). Perfectionism, academic motivation, and psychological adjustment: An integrative model. Personality and Social Psychology Bulletin, 31, 913-924. doi:10.1177/0146167204272298

Mościcki, E. K. (1997). Identification of suicide risk factors using epidemiologic studies. Psychiatric Clinics of North America, 20, 499-517. doi:10.1016/S0193-953X(05)70327-0

Niemiec, C. P., \& Ryan, R. M. (2009). Autonomy, competence, and relatedness in the classroom: Applying self-determination theory to educational practice. Theory and Research in Education, 7, 133-144. doi:10.1177/1477878509104318

Nikolai, J., Silva, P., \& Walters, S. (2017). Student and lecturer perspectives informing an academic support strategy to assist students in a medium-sized tertiary institution. Journal of the Association of Tertiary Learning Advisors Aotearoa, 2, 1-18. doi:10.26473/atlaanz.2017.2.1/001

Osman, A., Bagge, C. L., Gutierrez, P. M., Konick, L. C., Kooper, B. A., \& Barrios, F. X. (2001). The Suicidal Behaviors Questionnaire-Revised (SBQ-R): Validation with clinical and nonclinical samples. Assessment, 5, 443-454. doi:10.1177/107319110100800409

Pisarik, C. T. (2009). Motivational orientation and burnout among undergraduate college students. College Student Journal, 43, 1238-1252. 
Pittman, L. D., \& Richmond, A. (2008). University belonging, friendship quality, and psychological adjustment during the transition to college. The Journal of Experimental Education, 76, 343-362. doi:10.3200/JEXE.76.4.343-362

Pryor, J. H., Hurtado, S., Sharkness, J., \& Kom, W. S. (2008). The American freshman: National norms for fall 2007. Los Angeles, CA: Higher Education Research Institute.

Ross, S. E., Niebling, B. C., \& Heckert, T. M. (1999). Sources of stress among college students. College Student Journal, 33, 312-317.

Rowe, C. A., Walker, K. L., Britton, P. C., \& Hirsch, J. K. (2013). The relationship between negative life events and suicidal behavior: Moderating role of basic psychological needs. Crisis: The Journal of Crisis Intervention and Suicide Prevention, 34, 233-241. doi:10.1027/0227-5910/a000173

Ryan, R. M., \& Deci, E. L. (2000a). The darker and brighter sides of human existence: Basic psychological needs as a unifying concept. Psychological Inquiry, 11, 319-338. doi:10.1207/S15327965PLI1104_03

Ryan, R. M., \& Deci, E. L. (2000b). Intrinsic and extrinsic motivations: Classic definitions and new directions. Contemporary Educational Psychology, 25, 54-67. doi:10.1006/ceps.1999.1020

Scheel, M. J., Madabhushi, S., \& Backhaus, A. (2009). The academic motivation of at-risk students in a counseling prevention program. The Counseling Psychologist, 37, 1147-1178. doi:10.1177/0011000009338495

Schiffrin, H. H., Liss, M., Miles-McLean, H., Geary, K. A., Erchull, M. J., \& Tashner, T. (2014). Helping or hovering? The effects of helicopter parenting on college students' well-being. Journal of Child and Family Studies, 23, 548-557. doi:10.1007/s10826-013-9716-3

Schwartz, L. J., \& Friedman, H. A. (2009). College student suicide. Journal of College Student Psychotherapy, 23, 78-102. doi:10.1080/87568220902743058

Smith, T. R., Langenbacher, M., Kudlac, C., \& Fera, A. G. (2013). Deviant reactions to the college pressure cooker: A test of general strain theory on undergraduate students in the United States. International Journal of Criminal Justice Sciences, 8, 88-104. Retrieved from http://www.sascv.org/ijcjs/ pdfs/smithetalijcjs2013vol8issue2.pdf

Stahl, S. M. (2002). The psychopharmacology of energy and fatigue. The Journal of Clinical Psychiatry, 63, 7-8. doi:10.4088/JCP.v63n0102

Tomiyama, A. J., \& Mann, T. (2008). Cultural factors in collegiate eating disorder pathology: When family culture clashes with individual culture. Journal of American College Health, 57, 309-314. doi:10.3200/ JACH.57.3.309-314

Urdan, T., \& Schoenfelder, E. (2006). Classroom effects on student motivation: Goal structures, social relationships, and competence beliefs. Journal of School Psychology, 44, 331-349. doi:10.1016/j. jsp.2006.04.003

Vallerand, R. J., Pelletier, L. G., Blais, M. R., Briere, N. M., Senecal, C., \& Vallieres, E. F. (1992). The Academic Motivation Scale: A measure of intrinsic, extrinsic, and amotivation in education. Educational and Psychological Measurement, 52, 1003-1017. doi:10.1177/0013164492052004025

Vallerand, R. J., Pelletier, L. G., Blais, M. R., Brière, N. M., Senecal, C., \& Vallieres, E. F. (1993). On the assessment of intrinsic, extrinsic, and amotivation in education: Evidence on the concurrent and construct validity of the Academic Motivation Scale. Educational and Psychological Measurement, 53, 159-172. doi:10.1177/0013164493053001018

Van Orden, K. A., Witte, T. K., Cukrowicz, K. C., Braithwaite, S. R., Selby, E. A., \& Joiner, T. E., Jr. (2010). The interpersonal theory of suicide. Psychological Review, 117, 575-600. doi:10.1037/a0018697

Walton, G. M., \& Cohen, G. L. (2011). A brief social-belonging intervention improves academic and health outcomes of minority students. Science, 331, 1447-1451. doi:10.1126/science.1198364

Wang, K. T. (2012). Personal and family perfectionism of Taiwanese college students: Relationships with depression, self-esteem, achievement motivation, and academic grades. International Journal of Psychology, 47, 305-314. doi:10.1080/00207594.2011.626050

Westefeld, J. S., Button, C., Haley, J. J., Kettmenn, J. J., MacConnell, J., Sandil, R., \& Tallman, B. (2006). College student suicide: A call to action. Death Studies, 30, 931-956. doi:10.1080/07481180600887130 